\title{
Analysis of Current Reaction on Inverter-Switching to Detect Changes in Electrical Machine's High- Frequency Behavior
}

\author{
P. Nussbaumer, C. Santin, Th.M. Wolbank \\ Department of Electrical Drives and Machines \\ Vienna University of Technology, Austria \\ thomas.wolbank@tuwien.ac.at
}

\begin{abstract}
Adjustable Speed Drives (ASD) are the favorable choice in a fast increasing scope of applications. Although generally highly reliable, continuous condition monitoring of electrical drive systems becomes more and more important, as the adoption of such systems in safety critical applications, like x-bywire and in the so called more electric aircraft, increases. To fulfill the demand of high efficiency and good return on investment the different drive components are operated at or near their rated values. This fact and additional stress added due to high dynamic operation, fast switching of modern power electronics like IGBT lead to increased risk of drive breakdown. In many applications this is completely unacceptable and has to be compensated by preventive maintenance, fault tolerant operation or continuous condition monitoring and detection of faults in an early stage. A variable speed drive basically consists of three main components - the inverter, the cabling and the machine itself. These components - including their parasitic elements - form a complex impedance system. The proposed method evaluates the transient current reaction on inverter switching. A change in this reaction is correlated to a change in the mentioned complex impedance system, e.g. resulting from degradation of the insulation system. Application of highfrequency current sampling and further signal processing allow a very accurate monitoring of system parameters and even small changes in magnitude and location can be detected. Experimental results proof the applicability of the proposed condition monitoring technique.
\end{abstract}

\section{INTRODUCTION}

When it comes to the application of inverter-fed drives in safety critical devices, e.g. in the more electric aircraft or in industrial production lines with a high number of drive systems installed (e.g. steel or paper manufacturing), the demand for high reliability increases as a breakdown may lead to high economic losses. Preventive maintenance and continuous condition monitoring becomes a necessity for many applications.

The three major causes for electrical machine breakdown, according to literature, are bearing related faults with $\sim 50 \%$, stator related faults with $\sim 35 \%$ and rotor related faults with $\sim 10 \%$ [1] and [2]. As the proposed technique focuses on the detection of changes in the machine properties similar to the ones that occur during a degradation of the insulation system the focus will be on stator related faults. Around $70 \%$ out of the stator related faults are caused by turn and ground insulation failure.

Insulation failure is a slowly developing fault starting with degradation of the insulation leading to a turn-to-turn fault that speeds up and finally results in a severe ground fault and machine breakdown as described in [3]. As the last states are happening abrupt an early detection of insulation degradation is highly appreciated. As emphasized in [4] the major cause for insulation degradation is thermal stress besides electrical, mechanical and environmental strains. The high rise time of modern power electronics (for IGBT in the range of 50ns) leading to transient overvoltage further stress the insulation as analyzed e.g. in [3].

So far many different methods to detect insulation faults have been developed and presented in literature. Basically these can be categorized into offline and online procedures. The latter ones have the advantage that no disassembling or outtake of the drive system is required and are not limited to be applied only during general maintenance every three to six years [5]. However, most of the industrially accepted techniques are offline including the most promising one - the offline surge test [6].

The DC conductivity [7], the insulation resistance (IR) [8], $\mathrm{DC} / \mathrm{AC}$ HiPot [9] and polarization index (PI) [9] test are further offline insulation fault detection techniques. The Partial Discharge (PD) test is an industrially accepted online test, however only applicable for medium to high voltage machines and additional measurement equipment and high sophisticated evaluation algorithms are needed. This test is described e.g. in [10]. A summary and comparison of the different procedures can be found in [9].

Although industrially accepted, the above mentioned insulation fault detection techniques still have many disadvantages what led to the development of many other online insulation condition monitoring procedures. Only a short selection is mentioned in this introduction.

The online detection of turn faults can be employed by applying current signature analysis (CSA) as presented in [11] and [12]. An enhancement of the surge test to online application has been performed in [13]. The injection of a small high-frequency (hf) signal into the stator winding is suggested in [14] to detect a deterioration of the turn-to-turn insulation. The measurement and evaluation of the leakage current from conductor to ground to assess the machine's phase-to-ground insulation is presented in [3]. The calculation of the transient leakage inductance (influenced in case of a winding fault) by evaluation of the current reaction on 
voltage pulses applied by the voltage source inverter (VSI) can be used to detect a turn-to-turn fault as proposed in [15].

The requirements of the novel proposed technique are as follows. It has to be applicable online, the focus is on inverter-fed drive systems, no additional sensors except the ones already available for machine control shall be used and even a developing insulation fault shall be detectable before a turn-to-turn fault occurs. Although many different techniques for turn/insulation fault detection have been proposed so far, not all of the mentioned requirements can be reached. The focus on VSI-fed drive systems is of special interest as the short rise-time of the applied voltages in combination with the mismatch of the surge and machine impedance leads to transient overvoltage of twice the dc-link voltage and therefore additional stress for the machine's insulation system as investigated in [16] and [17].

The proposed technique is based on the detection of minor changes of the current's transient reaction on inverter switching due to a change in the machine's complex impedance system. Signal processing is applied and an insulation state indicator is developed to determine a degradation of the insulation in magnitude and location. Experimental results will show the method's general performance and sensitivity.

\section{FUNDAMENTALS OF THE PROPOSED CONDITION MONITORING METHOD}

Modern variable speed drives consist of three main components - the inverter, the cabling and the electrical machine itself. All these components are characterized by their electrical parameters like resistance and inductance per unit length for the cabling or stator resistance $r_{s}$, inductance $l_{s}$, etc. for the machine. However, also the parasitic components like capacitances phase-to-ground (cabling), winding-to-ground (machine), winding-to-winding (machine), turn-to-turn (machine) and the inverter's capacitive coupling to ground (between power semiconductors and grounded heat sink) influence the drive's electrical behavior especially in the high-frequency range. Many of the parasitic elements are influenced by the machine's or cable's insulation system and condition. Together with the other characteristic electrical parameters they form a complex impedance system.

If a steep voltage change e.g. through the VSI is applied the machine's high-frequency behavior is excited. Caused by the obvious mismatch of the drive's surge and machine impedance according to signal and systems theory this results in reflections of the voltage pulse at the machine terminals leading to transient overvoltage of up to twice or even four (in case of fast changes of the switching state) times the dclink voltage decaying as an exponentially damped sinusoid with frequency from several hundred $\mathrm{kHz}$ to the low $\mathrm{Mhz}$ range [17].

The described transient oscillation is visible not only in the reflected voltage but also in the current reaction on the applied voltage pulse. The proposed method's basic idea is to analyze the characteristics of this high-frequency behavior visible in the current measurements. Changes in the highfrequency behavior may be the result e.g. of insulation system degradation as this effect will change a component (windingto-winding capacitance,...) in the complex impedance system. If such a change can be detected accurately even a developing fault can be identified.

As the proposed method is based on current measurements only, no additional sensors are required. Only sufficient resolution in time of the current sampling process has to be guaranteed to accurately analyze the high-frequency oscillation in the current signal.

\section{MEASUREMENT Procedure, APPLIED SignAL PROCESSING AND CALCULATION OF INSULATION STATE INDICATOR}

\section{A. Measurement Procedure and Applied Signal Processing}

As described before the proposed method's basic idea is to analyze the transient current reaction on a voltage pulse excitation. For inverter-fed drives this can be relatively easy realized by changing the inverter switching states (e.g. PWMexcitation) and measuring the current with sufficient resolution in time. The switching transition used in this investigation is from inactive ( $000 \ldots$ lower short circuit) to active $(001 \ldots+\mathrm{U}, 010 \ldots+\mathrm{V}$ or $100 \ldots+\mathrm{W})$. If three different switching transitions are applied and the current reaction is analyzed, it is possible to perform a detection of the fault location as described later on. The indicator used to detect an alteration of the machine's high-frequent behavior is a comparative value calculated between a reference measurement (healthy machine condition) and a later measurement. The detailed calculation of this indicator will be described in III. $B$.

The signal processing starts with the measurement of the current reaction on a voltage pulse as shown in Fig. 1. The depicted current signal is measured in phase $U$ and is the reaction on a change of the inverter switching state from lower short circuit $(000)$ to $+\mathrm{U}(001)$. The overshot and decaying high frequent oscillation is clearly visible for about $10 \mu \mathrm{s}$ after the switching instant at around $4 \mu \mathrm{s}$.

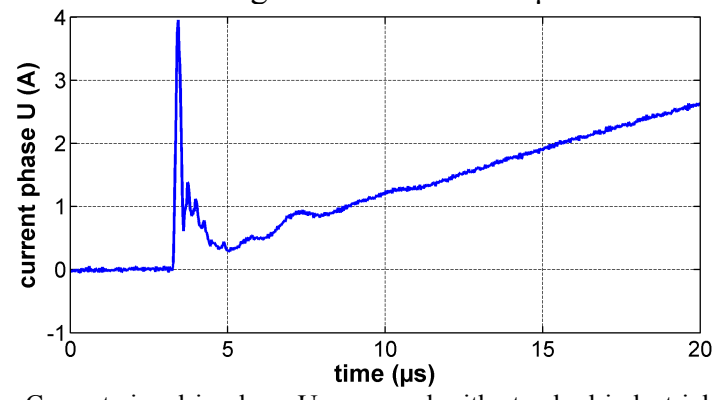

Fig. 1. Current signal in phase $U$ measured with standard industrial current sensors during switching of the VSI sampled with $40 \mathrm{MS} / \mathrm{s}$.

Henceforward the signal has a constant derivative depending on the machine's transient reactances. These reactances are usually modulated due to inherent asymmetries (e.g. slotting, saturation,...) and therefore depend on the rotor position. This position dependency is eliminated in a very easy way by subtracting the steady state current derivative after exact detection of the switching instant. The resulting 
signal is shown in Fig. 2.

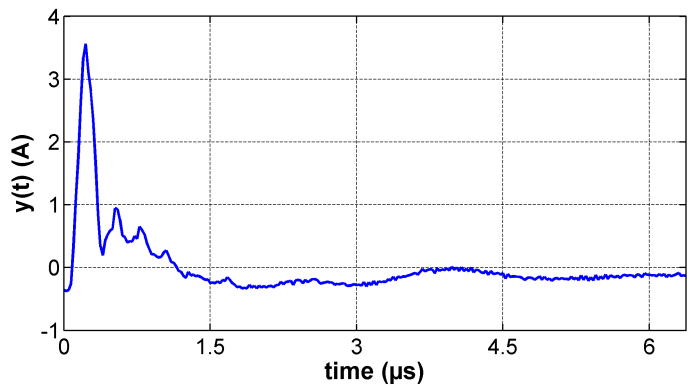

Fig. 2. Measured current reaction in phase $U$ to switching from inactive to active $(+\mathrm{U})$ after signal processing $y_{k}(t)$.

This signal is then transformed to the frequency domain by fast Fourier transform (FFT). To ensure that no dc-component is distracting the analysis a potential mean value is subtracted from the transformed signal as well. The amplitude spectrum of the Fourier-transformed signal in Fig. 2 is depicted in Fig. 3.

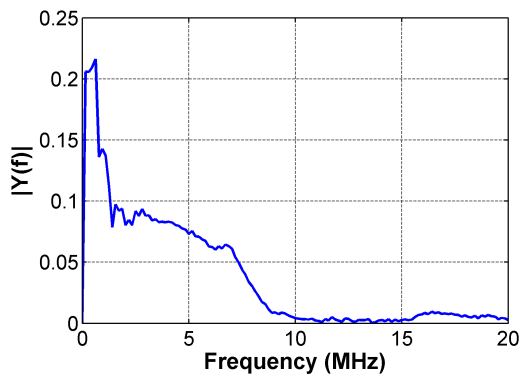

Fig. 3. Amplitude spectrum of measured current in phase $U$ resulting from switching (inactive to active $+\mathrm{U}$ ) after signal processing $Y_{k}(f)$.

The amplitude spectrum in Fig. 3 represents the reference trace that is compared to all later measurements and is recorded for the three different changes of switching states $(000 \rightarrow 001 ; 000 \rightarrow 010 ; 000 \rightarrow 100)$. For each switching state the current measurements and analyses are performed in the corresponding phase.

After the initial measurement to identify the reference trace (healthy condition) for every switching transition, the machine's step response on a switching transition (PWM) has to be measured for the three different excitations in the corresponding phase. The above described signal processing steps have to be carried out for each of the three measurements. A comparative value for each phase measurement between the amplitude spectrum of the reference measurement and the one of the condition measurement is calculated. Linear combination of the three measurements leads to a spatial representation of the insulation state and represents the spatial insulation state indicator (SISI), represented by a vector as described below in equation (6). A change in the complex impedance system in one of the three phases results in a deviation of the SISI from the origin. The direction of the deviation correlates with the location of the alteration the magnitude corresponds with the severity.

To improve accuracy the measurements can be repeated several times and statistical properties like mean or median values of the comparative value can be used as the insulation state indicator. As the applied voltage steps are in the range of some $\mu$ s only the measurement duration still remains low.

In the present investigation the number of measurements is chosen to 140. The mean trace of the healthy machine's amplitude spectra serves as the reference for the condition monitoring measurements.

\section{B. Calculation of Insulation State Indicator}

The selection and accurate calculation of the indicator to detect deviations from the reference measurements is a very important criterion for the proposed condition monitoring technique.

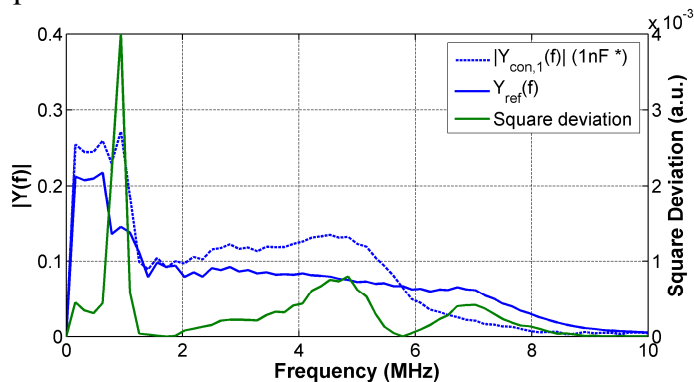

Fig. 4. Reference amplitude spectrum $Y_{\text {ref }}(f)$, amplitude spectrum of one measurement for inserted $1 \mathrm{nF}$ capacitor in parallel to the full winding of phase $\mathrm{U}\left|Y_{\text {con, } 1}(f)\right|$ and calculated square deviation between the two traces.

For the present investigation the Root Mean Square Deviation (RMSD) has been found to be a good choice. The definition of this comparative value is as follows

$\operatorname{RMSD}_{k}\left(x_{1}, x_{2}\right)=\frac{\sqrt{\sum_{i=1}^{n}\left(x_{1}(i)-x_{2, k}(i)\right)^{2}}}{n}$.

As mentioned before the mean trace of the healthy machine's amplitude spectrum serves as reference and is represented as $x_{1}$ in equation (1). This value is calculated according to

$x_{1}=Y_{\text {ref }}(f)=\frac{\sum_{k=1}^{m}\left|Y_{k}(f)\right|}{m}$

where $m$ is the number of measurements. In case of the present investigation $m$ is chosen to 140 . The index $k$ represents the consecutive number of the repeated measurements ([1...140] in present investigation). The index $i$ gives the frequency considered. The value of $n$ depends on the frequency resolution. In this investigation the analysis in the frequency domain $n$ results in 129 . The chosen window length equals to $6.4 \mu \mathrm{s}$ ( 256 sample at $40 \mathrm{MHz}$ sampling rate). The variable $x_{2, k}$ in equation (1) represents a single condition measurement after signal processing similar to the amplitude spectrum $Y_{c o n, k}$ depicted in Fig. 3.

$x_{2, k}=\left|Y_{c o n, k}(f)\right|$

With these equations an insulation state indicator (ISI) can be defined for changes of the switching state in the three different phases $p(\mathrm{U}, \mathrm{V}, \mathrm{W})$ and analysis of the corresponding phase current measurement as

$I S I_{p, k}=R M S D_{p, k}\left(x_{1}, x_{2}\right)=\frac{\sqrt{\sum_{i=1}^{n}\left(Y_{r e f}(f)-\left|Y_{c o n, p, k}(f)\right|\right)^{2}}}{n}$. 
The reference trace, a trace of a condition measurement where a $1 \mathrm{nF}$ capacitor is added in parallel to the full winding of phase $U$ and the corresponding square deviation are illustrated in Fig. 4. An increasing value of the ISI correlates with increasing change in the machine's high-frequency behavior (e.g. due to degradation of the insulation system). For each condition measurement $m$ insulation state indicators exist for each phase.

To increase accuracy statistical properties like mean or median value can be used to calculate one indicator for each phase $I S I_{p}$. For the present investigation the mean value is chosen.

$$
I S I_{p}=\frac{\sum_{k=1}^{m} I S I_{p, k}}{m}
$$

If the change in the high-frequency behavior is affecting only one phase, the indicator will increase only for this phase. By linear combination of the three ISI it is possible to identify the location of this asymmetry. Furthermore the effect of possibly disturbing influences like temperature and humidity occurring symmetrically in all three phases can be thus reduced. A novel spatial insulation state indicator (SISI) is created by such linear combination.

$$
S I S I=I S I_{U}+I S I_{V} \cdot e^{j \frac{2 \pi}{3}}+I S I_{W} \cdot e^{j \frac{4 \pi}{3}}
$$

\section{EXPERIMENTAL SETUP AND RESULTS}

\section{A. Experimental Setup and Fault Condition Realization}

To evaluate the proposed condition monitoring method a $5.5 \mathrm{~kW}, 2$-pole, squirrel-cage induction machine with tapped windings and an un-skewed rotor cage is used. Different taps in all three phases are accessible at the machine terminals and allow a realization of short circuit between single turns of the winding. Therefore short circuits can be realized without destruction of the test machine.

If instead of full short circuits a capacitor is inserted between two taps the machine's high-frequency behavior can be altered. In this way a replication of degraded insulation can be realized.

Fig. 5 shows a schematic overview of the machine's different parasitic elements like phase-to-ground $C_{p h \text {-gnd }}$, phase-to-phase $C_{p h-p h}$ and turn-to-turn $C_{t-t}$ capacitance. An additional capacitor inserted between the machine's terminal connection and the tap of the first turn is also depicted. Such an additionally inserted capacitance will be further denoted as fault capacitance $C_{\text {fault }}$.

The test machine's phase-to-ground $C_{p h \text {-gnd }}$ and phase-tophase $C_{p h-p h}$ capacitance have been determined to $1.71 \mathrm{nF}$ and $742 \mathrm{pF}$, respectively. The insertion of the fault capacitance in parallel to the capacitance of the healthy insulation results in an increase. This is in correlation with the investigations in [14].

Machine control and measurements are realized by a combination of real-time system and a powerful FPGA (National Instruments) programmable under LabVIEW for the PWM generation $(5 \mathrm{kHz})$ and data preprocessing. The current sampling is done with external $40 \mathrm{MHz} 16 \mathrm{bit}$ analogto-digital converters (ADC) communicating with the FPGA via data buffers (FIFOs).

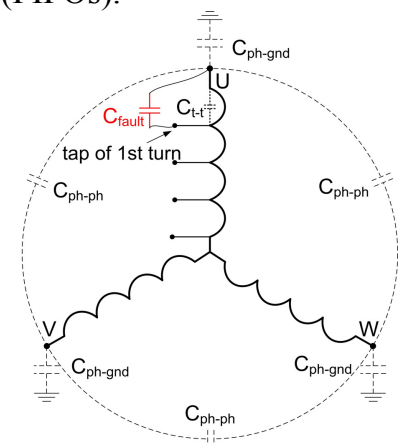

Fig. 5. Additional Capacitor $C_{\text {fault }}$ inserted between machine terminal connection of phase $U$ and tap of a turn in phase $U$.

To proof the proposed method's capability to detect a change in the machine's high-frequency behavior capacitors of different values and at different locations have been inserted.

\section{B. Experimental Results}

The experimental results in the following show the possibility to detect an alteration of the machine's highfrequency behavior in severity and location by calculation of the spatial insulation state indicator SISI. Measurements with various different capacitors $C_{\text {fault }}(200 \mathrm{pF}, 333 \mathrm{pF}, 500 \mathrm{pF}$ and $1 \mathrm{nF}$ ) inserted at different locations are compared. The capacitors have been inserted between different taps and in different phase windings. Furthermore the SISI of the healthy machine condition has been calculated. Moreover measurements have been carried out at standstill and rotating machine (no fundamental wave excitation).

To demonstrate the accuracy of the proposed condition monitoring method and proof the reproducibility of the insulation state detection a statistical analysis of the ISI has been carried out. Several different conditions have been realized and the ISI state has been recorded 140 times for each condition. A very convenient way to graphically depict the statistical properties of the calculated indicators is the socalled box plot. Such box plots have been created for the different realized conditions and are depicted in Fig. 6. All indicators have been calculated for phase $\mathrm{U}\left(I S I_{U}\right)$. The red horizontal line in the center of each box marks the median for the different ISI-values. The upper and lower limit represents the $75^{\text {th }}$ and $25^{\text {th }}$ percentile, respectively. A separation of the different conditions can be guaranteed if the notches of the different box plots don't overlap as in that case the true medians differ with $95 \%$ confidence. As can be seen for the analyzed machine conditions this criterion is fulfilled. The names (e.g. ' $U$ lnF full') used to identify the different conditions in figures and tables obey the following convention: location of insertion (e.g. $U$ for insertion in phase $\mathrm{U}$ ) and severity of applied change (value of inserted capacitor (e.g. $1 \mathrm{nF})$, insertion in parallel to 'full' or 'partial' $(\sim 50 \%)$ phase winding). 


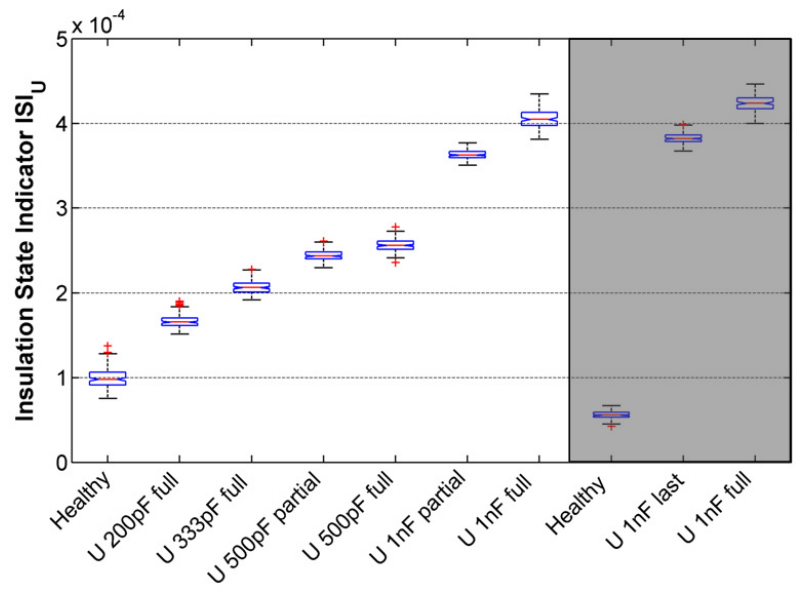

Fig. 6. Box plots of ISI calculated for phase U; different realizations of insulation state; measurements for standstill (left) and rotating machine (right, gray area).

The measurements denoted 'Healthy' in Fig. 6 have been both collected for healthy machine condition. One has been collected at standstill (left-hand-side), the other while the machine has been rotated (right-hand-side, gray area). It can be seen that both conditions are easily separable from the investigated machine condition with a small capacitor $(200 \mathrm{pF})$ inserted in parallel to the full winding of phase $U$ (' $U$ $200 \mathrm{pF}$ full'). If the SISI is regarded, as will be done in the following, the deviation of the measurements for same condition will be even smaller as only asymmetry is considered. As can be seen in Fig. 6 similar conditions that have been analyzed for rotating machine (gray area) or at standstill have very close indicators.

For a fault occurring in a phase different from the analyzed one, no alteration in the insulation state indicator is detectable. That is why the detection procedure has been expanded to the calculation of the spatial insulation state indicator SISI.

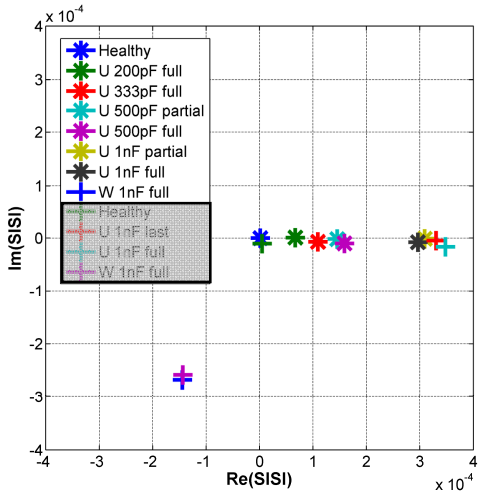

Fig. 7. Spatial insulation state indicator (SISI) for different investigated machine conditions.

Fig. 7 shows the calculated spatial insulation state indicator for different realized alteration of the machine's highfrequency behavior in phase $\mathrm{U}$ or $\mathrm{W}$ for machine at standstill and rotating machine (gray area). Table I summarizes the calculated spatial insulation state fault indicators. As the linear combination of the three different phase insulation state indicators according to equation (6) leads to a complex spatial insulation state indicator an alteration of the high-frequency behavior can be analyzed in location and severity. The magnitude of the spatial insulation state indicator corresponds with the severity, the angle with the location.

The results depicted in Fig. 7 and Table I show a very good separation of the different investigated conditions. The fault conditions realized in phase $U$ all lie in direction of the real axis with increasing magnitude for increasing size of the inserted capacitor. Whereas for the two conditions realized in direction of phase $\mathrm{W}$ the SISI lies $240^{\circ}$ shifted from the real axis equaling the direction $\mathrm{W}$. This proofs excellent separation of the fault location.

TABLE I

SPATIAL INSULATION STATE INDICATORS SISI GAINED AT DIFFERENT MACHINE CONDITIONS

\begin{tabular}{|c|c|c|}
\hline & Fault condition & $\operatorname{SISI}\left(\cdot 10^{-6}\right)$ \\
\hline \multirow{8}{*}{ 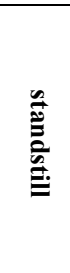 } & Healthy & $1.4532 \cdot \mathrm{e}^{-\mathrm{j} \cdot 3.48^{\circ}}$ \\
\hline & U 200pF full & $66.963 \cdot \mathrm{e}^{\mathrm{j} \cdot 0.89^{\circ}}$ \\
\hline & U 333pF full & $109.49 \cdot \mathrm{e}^{-\mathrm{j} \cdot 3.72^{\circ}}$ \\
\hline & U 500pF partial & $145.23 \cdot \mathrm{e}^{-\mathrm{j} \cdot 0.74^{\circ}}$ \\
\hline & U 500pF full & $159.11 \cdot \mathrm{e}^{-\mathrm{j} \cdot 3.65^{\circ}}$ \\
\hline & U 1nF partial & $309.04 \cdot \mathrm{e}^{-\mathrm{j} \cdot 0.22^{\circ}}$ \\
\hline & U 1nF full & $296.39 \cdot \mathrm{e}^{-\mathrm{j} \cdot 1.50^{\circ}}$ \\
\hline & W 1nF full & $303.88 \cdot \mathrm{e}^{-\mathrm{j} \cdot 118.37^{\circ}}$ \\
\hline \multirow{4}{*}{ 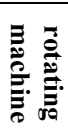 } & Healthy & $11.409 \cdot \mathrm{e}^{-\mathrm{j} \cdot 66.31^{\circ}}$ \\
\hline & U 1nF partial & $330.33 \cdot \mathrm{e}^{\mathrm{-j} \cdot 0.86^{\circ}}$ \\
\hline & U 1nF full & $348.08 \cdot \mathrm{e}^{-\mathrm{j} \cdot 2.72^{\circ}}$ \\
\hline & W 1nF full & $295.42 \cdot \mathrm{e}^{-\mathrm{j} \cdot 119.07^{\circ}}$ \\
\hline
\end{tabular}

If the magnitude of the different spatial insulation state indicators is compared one can see that for same condition the values a relatively close, independent of location of the fault. For the fault conditions where a $1 \mathrm{nF}$ capacitor is inserted in parallel to the full winding in two different phases (e.g. ' $U$ $1 \mathrm{nF}$ full' and ' $\mathrm{W} 1 \mathrm{nF}$ full) the magnitudes of SISI for the fault in phase $U$ and $W$ are $296.39 \cdot 10^{-6}$ and $303.88 \cdot 10^{-6}$, respectively.

However, one inconsistency can be seen when looked at the $1 \mathrm{nF}$ capacitors inserted in phase $\mathrm{U}$ between different taps. The values in this case are also relatively close no matter if e.g. inserted in parallel to the full or partial winding. The magnitudes of SISI for these two conditions equal $296.39 \cdot 10^{-6}$ (' $\mathrm{U} 1 \mathrm{nF}$ full') and $309.04 \cdot 10^{-6}$ (' $\mathrm{U} 1 \mathrm{nF}$ partial'), respectively. As the whole spectrum is analyzed in the proposed signal processing this difference can result from changes in different frequency ranges of the amplitude spectrum for the different conditions. This has to be further investigated and possible separation of the described conditions has to be analyzed for an indicator that takes only certain frequency ranges into account. This analysis is currently under development and is a very important task to improve the proposed novel condition monitoring technique.

As already described in [14] the machine's high-frequency behavior can be analyzed with a LCR-meter also. Such an analysis carried out with a Bode 100 by OMICRON Lab can be seen in Fig. 8. The blue trace shows the magnitude plot of the impedance measurement for the healthy machine whereas the green trace shows the measurement for a condition, where a $1 \mathrm{nF}$ capacitor was inserted in parallel to the full winding of phase U. Both measurements where performed in phase U. It can be clearly seen that for low frequencies the impedances 
for both conditions are the same. However the high-frequency behavior is significantly changed.

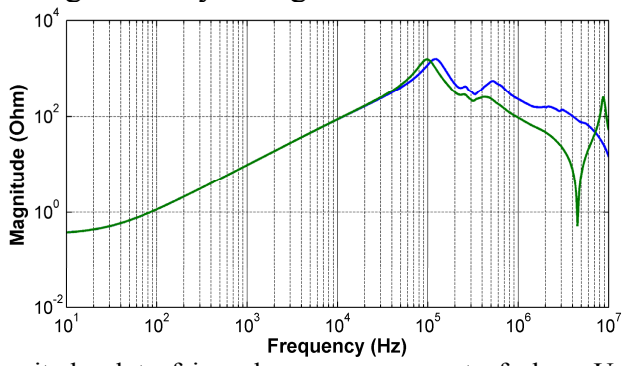

Fig. 8. Magnitude plot of impedance measurement of phase $\mathrm{U}$; frequency sweep $10 \mathrm{~Hz}-10 \mathrm{MHz}$, healthy machine (blue) and $1 \mathrm{nF}$ inserted in parallel to the full winding of phase $\mathrm{U}$ (green).

For the measurements shown in Fig. 8 a RMSD-value between the healthy (blue) trace and a condition measurement (e.g. green trace) can be calculated according to equation (1) as well. The results for some selected conditions have been summarized in Table II. A comparison between the $\mathrm{ISI}_{\mathrm{U}^{-}}$ value and the RMSD-value as just described can also be found in Table II. The indicators have been normalized to the value resulting from measurements with a $1 \mathrm{nF}$ capacitor inserted for better comparability. As can be seen the SISI proposed offers accuracy comparable to that of an industrial laboratory LCR meter.

TABLE II

COMPARISON OF RMSD-VALUE OF LCR-METER MEASUREMENTS AND ISI I

\begin{tabular}{|c|c|c|}
\hline $\begin{array}{ll}\text { Fault condition } & \text { Indicator } \\
\end{array}$ & $\begin{array}{c}\text { RMSD } \\
\text { LCR-meter }\end{array}$ & $\mathbf{I S I}_{\mathbf{U}}$ \\
\hline U 333pF full & 0.5381 & 0.5197 \\
\hline $\mathrm{U} 500 \mathrm{pF}$ full & 0.7077 & 0.6425 \\
\hline $\mathrm{U} 1 \mathrm{nF}$ full & 1.000 & 1.000 \\
\hline
\end{tabular}

The focus of this paper is a novel machine condition monitoring technique that is able to detect changes in the machine's high-frequency behavior caused e.g. by degradation of the machine's insulation system. The method's basic idea is to analyze the transient reaction of the machine current on inverter switching (e.g. PWM-excitation). All drive components consist besides their characteristic components of parasitic components that influence the machine's high-frequency behavior and lead - due to the mismatch of surge and machine impedance - to transient decaying oscillations following a change of the inverter switching state. This transient oscillation is visible in the measured current. Thus machine terminal voltage measurement can be avoided. By applying high-frequency sampling and further signal processing it is possible to create an insulation state indicator that enables to separate different high-frequency characteristics due to e.g. changes of the insulation capacitance. The signal processing is based on the comparison of the Fourier amplitude spectrum of a condition monitoring measurement to a reference measurement of the healthy machine. The applied comparative value is the Root Mean Square Deviation. By linear combination of calculated insulation state indicators to a spatial insulation state indicator it is possible to detect an alteration of the machine's highfrequency behavior in severity and location. Several different conditions have been analyzed and their accurate separation has been proven by experimental measurements on an induction machine.

\section{ACKNOWLEDGMENT}

The work to this investigation was supported by the Austrian Science Fund (FWF) under grant number P23496N24. The authors want to thank National Instruments Austria for the donation to finance the measurement hardware and especially DI Günther Stefan for the generous support.

\section{REFERENCES}

[1] IEEE Committee Report; "Report of large motor reliability survey of industrial and commercial installation, Part I," IEEE Transactions on Industry Applications, vol.21, no.4, pp.853-864, 1985.

[2] IEEE Committee Report; "Report of large motor reliability survey of industrial and commercial installation, Part II," IEEE Transactions on Industry Applications, vol.21, no.4, pp.865-872, 1985.

[3] Kim, H.D.; Yang, J; Cho, J.; Lee, S.B.; Yoo, J.-Y.; “An Advanced Stator Winding Insulation Quality Assessment Technique for Inverter-Fed Machines," IEEE Trans. on Ind. Appl., vol.44, no.2, pp.555-564, 2008.

[4] Grubic, S.; Aller, J.M.; Bin Lu; Habetler, T.G.; "A Survey on Testing and Monitoring Methods for Stator Insulation Systems of Low-Voltage Induction Machines Focusing on Turn Insulation Problems," IEEE Trans. on Industrial Electronics, vol.55, no.12, pp.4127-4136, 2008.

[5] Younsi, K.; Neti, P.; Shah, M.; Zhou, J.Y.; Krahn, J.; Weeber, K.; Whitefield, C.D.; "On-line capacitance and dissipation factor monitoring of AC stator insulation," IEEE Trans. on Diel. and El. Ins., vol.17, no.5, pp.1441-1452, 2010

[6] Wiedenbrug, E.; Frey, G.; Wilson, J.; "Impulse testing and turn insulation deterioration in electric motors," Annual Pulp and Paper Industry Technical Conference, pp. 50- 55, 2003.

[7] Schump, D.E.; "Testing to assure reliable operation of electric motors," Industry Applications Society 37th Annual Petroleum and Chemical Industry Conference, pp.179-184, 1990.

[8] Stone, G.C.; "Recent important changes in IEEE motor and generator winding insulation diagnostic testing standards," IEEE Transactions on Industry Applications, vol.41, no.1, pp. 91-100, 2005.

[9] Stone, G.C.; Boulter, E.A.; Culbert, I. and Dhirani, H.; "Electrical insulation for rotating machines - Design, Evaluation, Aging, Testing, and Repair," IEEE Press, John Wiley \& Sons, 2004.

[10] Stone, G.C.; Sedding, H.G.; Costello, M.J.; "Application of partial discharge testing to motor and generator stator winding maintenance," IEEE Trans. on Industry Applications, vol.32, no.2, pp.459-464, 1996.

[11] Joksimovic, G.M.; Penman, J.; "The detection of inter-turn short circuits in the stator windings of operating motors," IEEE Transactions on Industrial Electronics, vol.47, no.5, pp.1078-1084, 2000.

[12] Nandi, S.; Toliyat, H.A.; "Novel frequency-domain-based technique to detect stator interturn faults in induction machines using stator-induced voltages after switch-off," IEEE Trans. on Ind. Appl., vol.38, no.1, pp.101-109, 2002

[13] Grubic, S.; Habetler, T.G.; Restrepo, J.; “A new concept for online surge testing for the detection of winding insulation deterioration," Energy Conversion Congress and Exposition (ECCE), pp.2747-2754, 2010.

[14]Perisse, F.; Werynski, P.; Roger, D.; "A New Method for AC Machine Turn Insulation Diagnostic Based on High Frequency Resonances," IEEE Trans. on Diel. and El. Ins., vol.14, no.5, pp.1308-1315, 2007.

[15] Wolbank, T.M.; Wohrnschimmel, R.; "Transient electrical current response evaluation in order to detect stator winding interturn faults of inverter fed ac drives," Symposium on Diagnostics for Electric Machines, Power Electronics and Drives (SDEMPED), pp.1-6, 2001.

[16]Persson, E.; "Transient effects in application of PWM inverters to induction motors," IEEE Transactions on Industry Applications, vol.28, no.5, pp.1095-1101, 1992.

[17] Peroutka, Z.; "Requirements for insulation system of motors fed by modern voltage source converters," IEEE 35th Annual Power Electronics Specialists Conference, PESC, vol.6, pp. 4383- 4389, 2004. 